\title{
CONHECIMENTO, ATITUDES E PRÁTICAS DE ADOLESCENTES E PAIS SOBRE IMUNIZAÇÃO NA ADOLESCÊNCIA: REVISÃO SISTEMÁTICA
}

\section{Parents' and adolescents' knowledge, attitudes and practices related to immunization in adolescence: systematic review \\ Conocimiento, actitudes y prácticas de adolescentes y padres sobre la inmunización en la adolescencia: revisión sistemática}

\author{
Alisse Maria Chaves de Lima Peixoto (OrcID) \\ Universidade de Pernambuco - UPE - Camaragibe (PE) - Brasil \\ Paula Andréa de Melo Valença (Lattes) \\ Universidade de Pernambuco - UPE - Camaragibe (PE) - Brasil \\ Viviane Colares Soares de Andrade Amorim (OrcID) \\ Universidade de Pernambuco - UPE - Camaragibe (PE) - Brasil
}

\section{RESUMO}

Objetivo: Descrever os fatores associados aos conhecimentos, atitudes e práticas de adolescentes e seus pais frente à imunização na adolescência. Métodos: Revisão sistemática da literatura, realizada nos meses de março de 2017 a março de 2018 , por meio de três estratégias de busca no Portal CAPES e BVS, através dos descritores: adolescente; imunização; conhecimentos; atitudes e práticas em saúde; vacinação e controle de doenças transmissíveis. Os critérios de elegibilidade utilizados foram: artigos científicos publicados nos idiomas inglês, português e espanhol, nos anos de 2008 a 2018 . Após as buscas, incluíram-se 23 artigos na análise final. Resultados: Verificaram-se os seguintes fatores diretamente associados à imunização na adolescência: sexo; religião; raça/etnia; idade, local de residência; crenças sobre a eficácia e segurança da vacina; medo dos efeitos adversos; possuir convênio privado de saúde e recomendação pelos profissionais de saúde. No Brasil, alguns fatores diferem dos outros países, como a maior vacinação contra o HPV entre as meninas e contra a Hepatite B entre aqueles com menor renda per capita. Isto pode ser explicado porque o Brasil tem uma realidade econômica, social e um sistema de saúde diferente do que se observa nos demais países. Conclusão: Fatores sociodemográficos como sexo, raça/etnia, religião e idade foram associados ao conhecimento, atitudes e práticas acerca da imunização na adolescência. Fatores socioeconômicos e psicossociais, como insegurança, medo, preocupações com o custo da vacina, ter recebido recomendação e orientação sobre vacinação por um profissional de saúde também demonstraram associação.

Descritores: Adolescente; Vacinação; Imunização; Conhecimentos, Atitudes e Prática em saúde; Controle de Doenças Transmissíveis.

\begin{abstract}
Objective: To describe the factors associated with parents' and adolescents' knowledge, attitudes and practices related to immunization in adolescence. Methods: Systematic review of the literature carried out from March 2017 to March 2018 through three search strategies in the CAPES Portal and VHL using the descriptors: adolescent, immunization, health knowledge, attitudes and practices, vaccination and communicable disease control. The eligibility criteria used were: Scientific articles published in English, Portuguese and Spanish from 2008 to 2018. After the searches, 23 articles were included in the final analysis. Results: The factors directly associated with immunization in adolescence were: sex, religion, race/ethnicity, age, place of residence, beliefs about vaccine efficacy and safety, fear of adverse effects, private health insurance and recommendation by health professionals. In Brazil, some factors differ from those found in other countries, such as higher rates of vaccination against HPV among girls and Hepatitis B among those with lower per capita income. This can be explained by the fact that Brazil has economic and social realities and a health system that differ from what is observed in other countries. Conclusion: Socio-demographic factors such as sex, race/ethnicity, religion and age were associated with knowledge, attitudes and practices related to immunization in adolescence. Socioeconomic and psychosocial factors, such as insecurity, fear, concerns about the cost of the vaccine, having received advice and guidance on vaccination by a health professional were also associated.
\end{abstract}

Descriptors: Adolescent; Vaccination; Immunization; Health Knowledge, Attitudes and Practice; Communicable Disease Control. 


\section{RESUMEN}

Objetivo: Describir los factores asociados con los conocimientos, actitudes y prácticas de adolescentes y sus padres sobre la inmunización en la adolescencia. Métodos: Revisión sistemática de la literatura realizada en los meses entre marzo de 2017 y marzo de 2018 a través de tres estrategias de búsqueda en el Portal CAPES y BVS y con los descriptores adolescente; inmunización; conocimientos; actitudes e prácticas en salud; vacunación y control de enfermedades trasmisibles. Los criterios de elegibilidad utilizados fueron: artículos científicos publicados en los idiomas inglés, portugués y español en los años entre 2008 y 2018. Se han incluido 23 artículos en el análisis final. Resultados: Se verificaron los siguientes factores directamente asociados con la inmunización en la adolescencia: el sexo, la religión; la raza/etnia; la edad; el local de vivienda; las creencias sobre la eficacia y la seguridad de la vacuna; los miedos de los efectos adversos; el hecho de tener seguro privado de salud y recomendación de los profesionales sanitarios. En Brasil algunos factores son distintos de otros países como, por ejemplo, la mayor vacunación contra el Virus del Papiloma Humano (VPH) entre las niñas y contra la Hepatitis B entre aquellos con menor renta per cápita. Eso se puede explicar porque Brasil tiene una realidad económica, social y un sistema de salud distinto de los demás países. Conclusión: Los factores sociodemográficos como el sexo, la raza/etnia, la religión y la edad se asociaron con el conocimiento, las actitudes y las prácticas de la inmunización en la adolescencia. Los factores socioeconómicos y psicosociales como la inseguridad, el miedo, las preocupaciones con el coste de la vacuna y el hecho de haber recibido recomendación y orientación sobre vacunación por un profesional sanitario también demostraron asociación.

Descriptores: Adolescente; Vacunación; Inmunización; Conocimientos, Actitudes y Práctica en Salud; Control de Enfermedades Transmisibles.

\section{INTRODUÇÃO}

A adolescência sofre influência de diversos fatores, sociais, psicológicos, ambientais e econômicos, importantes para a formação do adolescente enquanto ser social. Apesar dos esforços governamentais visarem à atenção à saúde do adolescente, pode-se observar a presença de um perfil de morbimortalidade que poderia ser evitado através de medidas de promoção e prevenção da saúde. Dentre essas medidas de prevenção, a imunização através das vacinas constitui uma forma eficaz na prevenção de doenças imunopreveníveis ${ }^{(1)}$.

Dentre as vacinas recomendadas para os adolescentes, o Centers for Disease Control and Prevention (CDC) recomenda: influenza, dTpa (difteria, tétano e pertussis), HPV (Papiloma Vírus Humano) e meningocócica quadrivalente ${ }^{(2)}$. No Brasil, a vacina contra o HPV é disponibilizada pelo SUS para meninas de 9 a 15 anos de idade e para meninos de 11 a 15 anos de idade. Já a vacina meningocócica $C$, para adolescentes de 12 a 13 anos ${ }^{(3,4)}$. Além dessas duas citadas anteriormente, existem: a vacina contra Hepatite B; tríplice viral; a dT (dupla adulto - difteria e tétano), que deve ser administrada a cada 10 anos; a Influenza, administrada anualmente e oferecida pelo SUS apenas para grupos de risco; e a vacina contra febre amarela, com administração para viajantes e residentes em áreas recomendadas pelo Ministério da Saúde ${ }^{(5)}$.

Apesar das recomendações e esforços governamentais para melhorar a adesão e cobertura vacinal entre os adolescentes, esses fatores sofrem influência de aspectos históricos, presentes desde o surgimento das primeiras vacinas no mundo, que receberam inúmeras críticas e pouca aceitabilidade entre a população. Dentre esses fatores históricos, está a preocupação em relação à segurança das vacinas, sendo maior que o reconhecimento dos benefícios que a proteção através da vacinação pode trazer ${ }^{(6)}$.

Em relação à vacina contra o HPV, disponibilizada no Brasil desde 2014, a preocupação com a sua segurança, principalmente em relação aos eventos adversos, é um dos fatores que influenciam e justificam a sua baixa cobertura. Dado estatístico que comprova essa baixa adesão é que cerca de 5,5 milhões de meninas brasileiras têm o esquema vacinal contra o HPV incompleto ${ }^{(7)}$.

A articulação entre o conhecimento científico e o popular acerca da imunização é fundamental, visto que as crenças podem influenciar na atitude frente à vacinação. Nesse sentido, pode-se perceber que ações de educação em saúde, inclusive no ambiente escolar, ajudariam na adoção de boas práticas. Assim, é necessário conhecer quais fatores influenciam mais fortemente a imunização na adolescência, para que haja o direcionamento das ações de promoção da saúde ${ }^{(8)}$.

O objetivo deste estudo foi descrever os fatores associados aos conhecimentos, atitudes e práticas de adolescentes e seus pais frente à imunização na adolescência. 


\section{MÉTODOS}

Revisão sistemática da literatura, realizada de março de 2017 a março de 2018, por meio de busca no Portal de Periódicos da Coordenação de Aperfeiçoamento de Pessoal de Nível Superior (CAPES) e na Biblioteca Virtual em Saúde (BVS).

Utilizaram-se três estratégias de busca: a primeira, no Portal CAPES, através dos descritores: 1- vacinação AND adolescente. A segunda estratégia foi realizada na BVS (MEDLINE e LILACS), através dos descritores: 2- adolescente AND vacinação AND controle de doenças transmissíveis; e a terceira estratégia: 3- adolescente AND imunização AND conhecimentos, atitudes e práticas em saúde. Considerou-se a seguinte pergunta condutora: Quais os fatores associados aos conhecimentos, atitudes e práticas de adolescentes e seus pais sobre vacinação na adolescência?

Utilizaram-se como critérios de elegibilidade: artigos científicos publicados nos idiomas inglês, português e espanhol, nos anos de 2008 a 2018. Consideraram-se os critérios de inclusão: artigos científicos realizados na faixa etária de 10 a 19 anos e que respondessem à pergunta condutora; e os critérios de exclusão: artigos que não apresentassem resumo, artigos de revisão, pesquisas qualitativas, estudos de validação de questionário e artigos cujo objeto de estudo não foi o adolescente e/ou seus pais.

Por meio das três estratégias de busca, obtiveram-se 1912 artigos, totalizando 1534 após a aplicação dos filtros. Após a leitura dos títulos e resumos, 50 artigos foram eleitos e lidos na íntegra. Destes, 23 compuseram a amostra final da revisão. Na Figura 1 pode-se observar a síntese do processo de busca e seleção dos artigos. A seleção dos artigos ocorreu de modo independente por dois pesquisadores, sendo as distinções referentes à seleção dos artigos que iriam para análise, discutidas de modo conjunto pelos pesquisadores.

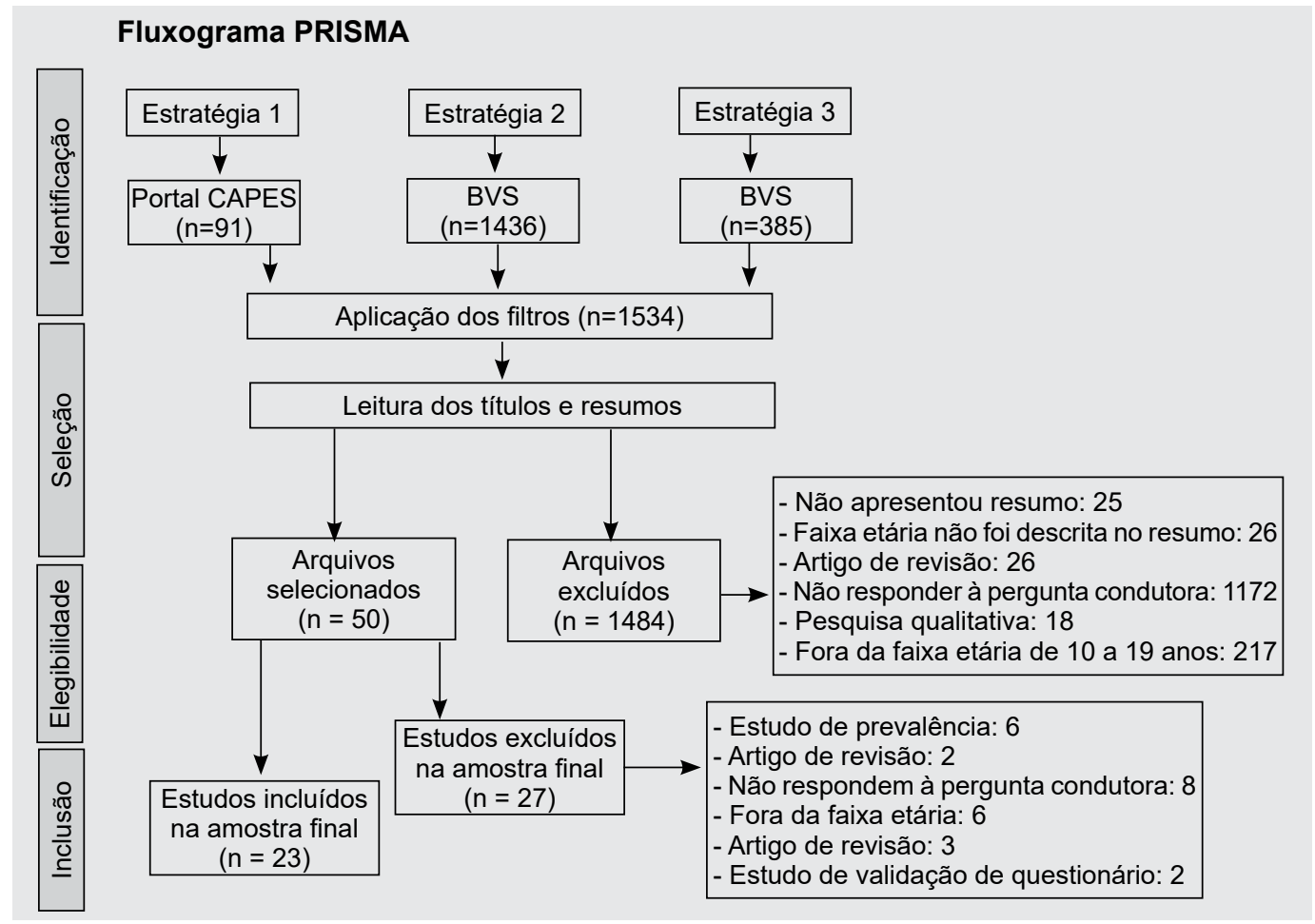

Figura 1 - Fluxograma de seleção dos estudos.

Para analisar a associação entre os fatores pesquisados e o conhecimento, atitudes e práticas frente à imunização, consideraram-se os valores de Odds ratio (OR), razão de prevalência (RP) e o valor de $p$ através do teste do qui-quadrado.

\section{RESULTADOS}

Os 23 artigos que compuseram a análise foram publicados nos anos de 2009 a 2017, a maioria no idioma inglês, havendo apenas dois em português. Grande parte dos estudos era realizada nos Estados Unidos (oito), embora outros países de origem também tenham sido encontrados: Brasil, Holanda, Itália, Grécia, China, Turquia, Bélgica, Índia e Suécia. O tipo de estudo mais prevalente foi o transversal, presente em 22 artigos, e a população-alvo mais frequente, os adolescentes (Quadro I). 
Quadro I - Distribuição dos artigos analisados por população-alvo, autor/ano, amostra, faixa etária, país.

\begin{tabular}{|c|c|c|c|c|}
\hline População-alvo & Autor (Ano) & $\begin{array}{c}\text { Amostra } \\
n\end{array}$ & Faixa etária & País \\
\hline \multirow{9}{*}{ Pais de adolescentes } & Zhang et al., 2013(9) & 2895 & $11-17$ & China \\
\hline & Gilkey et al., 2016(10) & 1495 & $11-17$ & Estados Unidos \\
\hline & Coyne-Beasley et al., $2013^{(11)}$ & 1281 & $11-17$ & Estados Unidos \\
\hline & Mukherjee et al., 2015(12) & 778 & $11-15$ & Índia \\
\hline & Seven et al., $2015^{(13)}$ & 368 & $10-13$ & Turquia \\
\hline & Reiter et al., 2014(15) & 2786 pais de meninas & $13-17$ & Estados Unidos \\
\hline & Bianco et al., 2014(14) & 566 pais meninos & $10-14$ & Itália \\
\hline & Taylor et al., 2014(17) & 758 pais de meninos & $11-17$ & Estados Unidos \\
\hline & Donahue et al., 2014(16) & 779 pais de meninos & $11-17$ & Estados Unidos \\
\hline \multirow{4}{*}{$\begin{array}{l}\text { Pares } \\
\text { pais/filhos }\end{array}$} & Kester et al., 2013(28) & 501 mães e meninas & $14-17$ & Estados Unidos \\
\hline & Van Keulen et al., 2014(26) & 642 meninas e 952 mães & $13-14$ & Holanda \\
\hline & Shao et al., $2015^{(31)}$ & 101 meninos e 35 pais & $13-19$ & Estados Unidos \\
\hline & Choi et al., 2014(29) & 2017 mães e 2167 meninas & $11-18$ & China \\
\hline \multirow{10}{*}{ Adolescentes } & Francisco et al., $2015^{(23)}$ & 702 & $11-19$ & Brasil \\
\hline & Friedrich et al., $2016^{(18)}$ & 390 & $11-18$ & Brasil \\
\hline & Kreuger et al., $2017^{(20)}$ & 390 & $12-17$ & Brasil \\
\hline & Gottvall et al., 2009(19) & 608 & $14-19$ & Suécia \\
\hline & Vaidakis et al, $2017^{(21)}$ & 4507 & 17 & Grécia \\
\hline & Devroey et al., 2013(22) & 186 & $14-17$ & Bélgica \\
\hline & Mollers et al., 2014(27) & 2989 meninas & $16-17$ & Holanda \\
\hline & Reiter et al., $2013^{(30)}$ & 1951 meninas & $14-17$ & Estados Unidos \\
\hline & Giambi et al., 2014(24) & 1738 meninas & $16-17$ & Itália \\
\hline & Bowyer et al., 2014(25) & 650 meninas & $16-18$ & Inglaterra \\
\hline
\end{tabular}

Oito artigos abordam a imunização apenas em adolescentes do sexo feminino, quatro apenas do sexo masculino e onze em adolescentes de ambos os sexos. Nas pesquisas que incluíram os conhecimentos, atitudes e práticas dos pais, verificou-se uma maior participação das mães ${ }^{(9-17)}$. Apesar da busca considerar a imunização de forma geral, dos vinte e três trabalhos, dezenove artigos pesquisaram apenas a vacina contra o HPV.

Observou-se associação de sexo com o conhecimento sobre vacinação, evidenciando que os adolescentes do sexo masculino tinham menos conhecimento sobre a vacina do HPV do que as adolescentes ${ }^{(13,18-20)}$. As áreas em que residem os adolescentes também puderam ser associadas, uma vez que adolescentes moradores de áreas urbanas tinham mais conhecimento sobre o tema ${ }^{(21)}$. Outros fatores associados ao conhecimento sobre a vacina do HPV se referiam: ao alto nível educacional dos pais, à recepção de informações através dos profissionais de saúde, aos pais de adolescente com idades entre 16 e 17 anos, a estudar em escola privada e a ter convênio privado de saúde (Quadro II).

Quadro II - Distribuição dos artigos analisados segundo fatores associados ao maior conhecimento sobre imunização segundo autor/ano, odds ratio (OR), valor de significância $(p)$ e tipo de vacina.

\begin{tabular}{|c|c|c|c|c|}
\hline $\begin{array}{l}\text { Fatores associados ao maior } \\
\text { conhecimento sobre imunização }\end{array}$ & Autor/ano & OR & $\mathbf{p}$ & Tipo de vacina \\
\hline \multirow{2}{*}{ Alto nível educacional dos pais } & Bianco et al., 2014 & 1,71 & - & HPV \\
\hline & Giambi et al., $2014^{(24)}$ & 3,76 & - & HPV \\
\hline Recepção de informações através dos médicos & Bianco et al., 2014(14) & 1,71 & - & HPV \\
\hline Residir em área urbana & Vaidakis et al., $2017^{(21)}$ & 1,45 & - & HPV \\
\hline Pais de adolescentes de idade de 16 a 17 anos & Coyne-Beasley et al., $2013^{(11)}$ & 1,82 & - & \\
\hline Estudar em escola privada & Coyne-Beasley et al., $2013^{(11)}$ & 1,95 & - & Meningocócica \\
\hline Possuir convênio privado de saúde & Coyne-Beasley et al., $2013^{(11)}$ & 1,90 & - & \\
\hline \multirow{4}{*}{ Sexo feminino } & Seven et al., $2015^{(13)}$ & - & $<0,05$ & HPV \\
\hline & Friedrich et.al., $2016^{(18)}$ & - & 0,000 & HPV \\
\hline & Kreuger et.al., $2017^{(20)}$ & - & $<0,05$ & HPV \\
\hline & - & & $<0,001$ & HPV \\
\hline
\end{tabular}

HPV: Papiloma Vírus Humano; OR: odds ratio 
Os fatores associados às atitudes e práticas frente à imunização mais citados pelos estudos: sexo, religião, raça e etnia, renda mensal, alto nível educacional dos pais, conhecimento sobre a vacina, recomendação da vacinação por um profissional de saúde, ter convênio privado de saúde e custo da vacina (Quadro III).

Em relação a outras vacinas estudadas, como meningocócica, por exemplo, os fatores associados ao conhecimento e aceitabilidade não diferem da vacina contra o HPV ${ }^{(11,12,22,23)}$.

Quadro III - Distribuição dos artigos analisados segundo fatores associados a melhores atitudes e práticas frente à imunização segundo autor/ano, odds ratio $(\mathrm{OR})$, valor de significância $(\mathrm{p})$, razão de prevalência $(\mathrm{RP})$ e tipo de vacina.

\begin{tabular}{|c|c|c|c|c|c|}
\hline $\begin{array}{l}\text { Fatores associados a melhores atitudes } \\
\text { e práticas frente à imunização }\end{array}$ & Autor (ano) & OR & $\mathbf{p}$ & $\mathbf{R P}$ & Tipo de vacina \\
\hline \multirow{13}{*}{$\begin{array}{l}\text { Contato/recomendação de profissional } \\
\text { de saúde }\end{array}$} & Zhang et al., $2013^{(9)}$ & 2,47 & - & - & HPV \\
\hline & Gilkey et al., $2016^{(10)}$ & 9,31 & - & - & HPV \\
\hline & Coyne-Beasley et al., $2013^{(11)}$ & 3,03 & - & - & Meningocócica \\
\hline & Mukherjee et al., $2015^{(12)}$ & 2,34 & - & - & Vacinas no geral \\
\hline & Bianco et al., 2014(14) & 1,49 & - & - & HPV \\
\hline & Reiter et al., 2014(15) & 1,70 & - & - & HPV \\
\hline & Taylor et al., 2014(17) & 2,22 & - & - & HPV \\
\hline & Francisco et.al., $2015^{(23)}$ & - & - & 2,27 & Hepatite B \\
\hline & Bowyer et al., 2014(25) & - & 0,027 & - & HPV \\
\hline & Van Keulen et al., 2013(26) & - & $<0,001$ & - & HPV \\
\hline & Kester et al.,2013 ${ }^{(28)}$ & - & 0,013 & - & HPV \\
\hline & Reiter et al., $2013^{(30)}$ & 4,07 & - & - & HPV \\
\hline & Shao et al., $2015^{(31)}$ & 2,5 & - & - & HPV \\
\hline \multicolumn{6}{|l|}{ Religião } \\
\hline Hindu & Mukherjee et al., $2015^{(12)}$ & 1,00 & - & - & Vacinas no geral \\
\hline Cristã & Bowyer et al., 2014(25) & - & 0,002 & - & HPV \\
\hline Não ser protestante & Van Keulen et al., 2013(26) & - & $<0,001$ & - & HPV \\
\hline Católica & Mollers et al., 2014 27$)$ & 1,2 & - & - & HPV \\
\hline \multicolumn{6}{|l|}{ Etnia/raça } \\
\hline Não hispânica / afro-americana & Coyne-Beasley et al., $2013^{(11)}$ & 2,17 & - & - & Meningocócica \\
\hline Preta & Taylor et al., 2014(17) & 2,08 & - & - & HPV \\
\hline Branca & Kester et al.,2013(28) & - & $<0,001$ & - & HPV \\
\hline \multicolumn{6}{|l|}{ Sexo } \\
\hline Masculino & Seven et al., $2015^{(13)}$ & - & $<0,05$ & - & HPV \\
\hline Feminino & Kreuger et.al., $2017^{(20)}$ & - & $<0,05$ & - & HPV \\
\hline \multicolumn{6}{|l|}{ Renda mensal } \\
\hline Menor renda & Francisco et.al., $2015^{(23)}$ & - & - & 1,16 & Hepatite B \\
\hline Maior renda & Choi et al., 2014(29) & 1,63 & - & - & HPV \\
\hline \multirow{3}{*}{ Possuir convênio privado de saúde } & Mukherjee et al., $2015^{(12)}$ & 2,20 & - & - & Vacinas no geral \\
\hline & Reiter et al., 2014(15) & 3,00 & - & - & HPV \\
\hline & Reiter et al., $2013^{(30)}$ & 3,67 & - & - & HPV \\
\hline Alto nível educacional dos pais & Mollers et al., 2014(27) & 1,3 & - & - & HPV \\
\hline
\end{tabular}

HPV: Papiloma Vírus Humano; OR: odds ratio; RP: razão de prevalência

\section{DISCUSSÃO}

Dentre os fatores sociodemográficos associados ao conhecimento sobre vacinação, os adolescentes do sexo masculino apresentaram menor conhecimento sobre a vacina do HPV do que as adolescentes em estudos realizados na Grécia, na Suécia e também no Brasil(18-21). Em relação ao sexo dos pais dos adolescentes, na Turquia, as mães apresentaram mais conhecimento sobre a vacina do que os pais, entretanto, nesse estudo, ambos tinham um nível de conhecimento muito baixo ${ }^{(13)}$.

Esses achados evidenciam um maior conhecimento por parte dos indivíduos do sexo feminino, seja entre as adolescentes, seja entre as mães, o que, talvez, esteja relacionado a um maior interesse das mulheres por temas relacionados à saúde, tanto no Brasil como em outros países. 
Outro fator sociodemográfico apresentado em um dos estudos foi o local de residência, pois os adolescentes que residiam em áreas urbanas tinham mais conhecimento sobre a vacina contra o HPV(21), assim como os pais que tinham um alto nível educacional apresentaram maior conhecimento(14,"24.).

Em relação aos fatores associados à vacina meningocócica, aqueles pais de adolescentes que estudaram em escolas particulares e tinham convênio privado de saúde apresentaram melhor conhecimento sobre a vacina. Além disso, a idade também foi associada, pois os pais dos adolescentes mais velhos (16 a 17 anos) detinham mais conhecimento que os pais dos mais jovens ${ }^{(11)}$.

Sobre os fatores sociodemográficos, que apresentaram associação com a intenção e a prática de vacinar, a religião foi abordada de diferentes formas em vários estudos. Enquanto um demonstrou que houve uma maior cobertura vacinal entre as adolescentes de religião cristã, outro estudo, realizado na Holanda, apresentou que adolescentes que não eram protestantes tiveram maior intenção de serem vacinadas ${ }^{(25,26)}$. Ainda na Holanda, outro estudo demonstrou que as adolescentes vacinadas contra o HPV eram, em sua maioria, católicas e, em sua minoria, protestantes ${ }^{(27)}$. Por outro lado, na Índia verificou-se que a religião hindu foi mais associada à completude do calendário vacinal dos adolescentes, entretanto esse resultado pode ter ocorrido devido ao país onde o estudo foi realizado, no qual a maioria da população segue a religião hindu ${ }^{(12)}$.

A raça/etnia também foi associada a atitudes e práticas acerca da vacinação, de modo que a raça negra esteve relacionada a não vacinação contra o HPV em adolescentes ${ }^{(25,28)}$. Sob outra perspectiva, uma pesquisa realizada nos Estados Unidos com pais de adolescentes do sexo masculino demonstrou que pais da raça negra e da etnia hispânica tiveram uma maior iniciação do esquema vacinal nos seus filhos do que os pais brancos ${ }^{(17)}$.

O tamanho da amostra e as análises dos estudos citados acima foram semelhantes, sendo a única diferença em relação à caracterização da amostra. Enquanto os estudos que demonstraram a raça negra associada a não vacinação ocorreram em adolescentes do sexo feminino, o terceiro estudo que apresentou resultados contrários foi realizado com adolescentes do sexo masculino ${ }^{(17,25,28)}$.

Dessa forma, a raça pareceu influenciar diferentemente ambos os sexos. Os estudos que apresentam as adolescentes de raça branca como mais prováveis a terem recebido a vacina discutem que isso ocorre porque o acesso à vacina e ao conhecimento sobre o tema é maior entre essas pessoas, influenciado por fatores socioeconômicos. A discrepância entre os achados pode sugerir que as razões para não vacinação entre os sexos são diferentes, de forma que o nível de conhecimento e a condição socioeconômica parecem exercer menor influência entre os pais de adolescentes do sexo masculino ${ }^{(17,25,28)}$.

Fazer parte ou não de um convênio privado de saúde foi citado em estudos como tendo associação com a vacinação dos adolescentes. Isto acontece porque em vários países, como Estados Unidos, China, Índia e Turquia, a vacina contra o HPV e outras vacinas não são disponibilizadas gratuitamente pelo governo. A não disponibilidade gratuita pode dificultar a adesão à vacinação nesses países, principalmente se o custo da vacina for alto e/ou se os pais não tiverem condições econômicas ${ }^{(12,13,17)}$. Um estudo realizado em Hong Kong também confirmou esses resultados ao demonstrar que famílias com um maior rendimento mensal estavam associadas à maior aceitabilidade em relação à vacina ${ }^{(29)}$.

Por outro lado, no Brasil, observou-se que há uma maior prevalência de vacinação contra hepatite $B$ entre os adolescentes com menor renda familiar per capita. Isto pode sugerir que, devido à disponibilização gratuita dessa vacina pelo SUS, as pessoas com menores condições socioeconômicas têm melhor acesso à vacinação no Brasil do que nos demais países citados acima. Além disso, as pessoas mais favorecidas economicamente nesses países podem não se enxergar como pessoas vulneráveis ao adoecimento por doenças infectocontagiosas ${ }^{(23)}$.

Preocupações a respeito da segurança e eficácia e do medo dos eventos adversos foram associados a atitudes relacionadas à vacina contra o HPV e à vacinação de forma geral. Adolescentes e seus pais relataram que constituem uma barreira para a vacinação, algo que sugere que as informações divulgadas na mídia, em escolas e pelos governantes não têm sido suficientes para influenciar positivamente a atitude frente à imunização( ${ }^{(12,24-26, " 19,23)}$.

Outra fonte de informação que exerce forte influência na tomada de decisão dos pais sobre vacinar ou não seus filhos são os profissionais de saúde. Dez dos 23 estudos associaram a recomendação dada pelos médicos e demais profissionais para que vacinassem seus filhos com a atitude e a prática de vacinar. Esse achado reflete que o pouco conhecimento dos pais sobre a vacinação leva à necessidade de buscar informações e recomendações através dos profissionais de saúde. Tal fato sugere a forte influência que esses profissionais têm na adesão à vacinação. Dessa forma, é necessário que eles sejam estimulados e capacitados pelo Ministério da Saúde para recomendar a completude do calendário vacinal e, assim, promover a saúde dos adolescentes ${ }^{(11,12,14,17-20,29,30)}$. 
Em contraposição à associação positiva da recomendação médica com a vacinação, um estudo realizado nos Estados Unidos com 101 adolescentes do sexo masculino, de 13 a 19 anos, evidenciou que os que visitavam um médico e realizavam exames regularmente interessavam-se menos pela vacina contra o HPV, assim como os adolescentes que tinham uso constante de preservativo ${ }^{(31)}$. Por outro lado, em relação ao uso de preservativo, um estudo realizado na Holanda evidenciou que as adolescentes que usavam método contraceptivo tinham mais chances de terem recebido a vacina ${ }^{(27)}$. Destaca-se que as amostras nesses dois estudos diferem bastante: enquanto o primeiro foi composto por uma amostra não representativa da população de 101 adolescentes masculinos, o segundo avaliou 2.989 adolescentes do sexo feminino. Além do tamanho da amostra, o estudo com os adolescentes ocorreu em apenas uma clínica.

Esse mesmo estudo, com uma amostra não representativa, também demonstrou que os adolescentes que tiveram um diagnóstico anterior de infecção sexualmente transmissível e que apresentaram um aumento na quantidade de parceiros sexuais tinham maiores chances de ter interesse em receber a vacina ${ }^{(31)}$.

Uma barreira apontada pelos pais, em alguns estudos, e que foi associada à vacinação contra o HPV de suas filhas foi a crença de que a idade é muito precoce para a administração de uma vacina que protege contra uma infecção sexualmente transmissível, principalmente porque acreditam que elas ainda não iniciaram a sua vida sexual. Dessa forma, a percepção de risco é menor ${ }^{(24,26,30)}$.

Além dos pais, profissionais de saúde também indicam menos a vacina quanto menor for a idade. Esses achados sugerem que ainda não está totalmente claro para a população que a eficácia da vacina é maior quando ela é administrada antes da exposição ao vírus, ou seja, antes da iniciação sexual, por isso delimita-se a idade, para aumentar a proteção ao vírus ${ }^{(28)}$. Uma pesquisa realizada na Bélgica demonstrou que a baixa informação sobre a necessidade da vacina e a não recomendação médica constituem uma barreira para a vacinação(22).

Apesar de haver menos informações sobre a eficácia da vacina do HPV entre os adolescentes, a vacinação das adolescentes do sexo feminino é a que apresenta mais entraves, conforme evidenciado em um estudo realizado nos Estados Unidos. Diferenças entre a aceitação da atividade sexual de filhos do sexo masculino e feminino é o principal obstáculo para os pais. Isto ocorre porque esses pais sentem-se mais confortáveis para discutir sobre saúde sexual com os filhos do que com as filhas; assim, a iniciação da vida sexual da filha torna-se um "tabu", algo que contribui para uma baixa adesão à vacina contra o HPV(17).

Por outro lado, no Brasil, verificou-se que mais adolescentes do sexo feminino foram vacinadas contra o HPV do que os adolescentes ${ }^{(20)}$. Isto pode ser explicado pelo fato do SUS ter iniciado a disponibilização dessa vacina apenas para as adolescentes do sexo feminino e, somente em 2017, os adolescentes terem sido incluídos no Calendário Vacinal do Ministério da Saúde.

Por consequência de todos os fatores discutidos anteriormente, estudos realizados evidenciaram em seus resultados a baixa adesão dos adolescentes à vacinação de forma geral, demonstrando que menos da metade da amostra recebeu uma ou todas as doses da vacina. Esse resultado sugere a necessidade da criação de novas estratégias para promover a saúde do adolescente e a prevenção de doenças através da imunização(13,14,19,20-27).

Para orientar essas estratégias de promoção da saúde, é importante perceber quais grupos de pessoas têm menos conhecimento e menos adesão à prática de vacinar. Assim, as ações de educação em saúde podem estar voltadas com maior enfoque para os meninos e para aqueles residentes de áreas rurais, por exemplo. Além disso, também é importante promover a saúde fora dos muros dos serviços de saúde, com ações de conscientização e de divulgação de informações nas igrejas, nas escolas e em outros espaços de convivência social.

A principal limitação desta revisão consiste na metodologia adotada pelos artigos, pois a maioria foi do tipo transversal. Neste tipo de estudo, devido ao fato de todas as variáveis serem pesquisadas ao mesmo tempo, não se pode inferir causalidade, o que pode dificultar a interpretação dos resultados.

Frente aos resultados desta pesquisa, observa-se a necessidade de estratégias de capacitação e que promovam um maior envolvimento dos profissionais de saúde na imunização dos adolescentes, através de ações de educação em saúde com foco nos adolescentes e seus pais.

\section{CONCLUSÃO}

Fatores sociodemográficos (como sexo, raça/etnia, religião, idade, alto nível educacional dos pais), assim como os socioeconômicos e psicossociais (como medo, preocupações com o custo da vacina, ter convênio privado de saúde, estudar em escola privada), estiveram associados ao conhecimento sobre as vacinas, à intenção de vacinar e à conclusão do esquema e calendário vacinal. O fator associado a melhores atitudes e práticas acerca da 
imunização mais citado em quase todos os estudos foi a recomendação da vacinação por um médico ou profissional de saúde. Dessa forma, pode-se considerar que esses profissionais são capazes de influenciar positivamente a cobertura vacinal dos adolescentes.

\section{CONTRIBUIÇÕES}

Alisse Maria Chaves de Lima Peixoto contribuiu na elaboração e no delineamento do estudo; na aquisição, na análise e na interpretação de dados; e na redação e/ou revisão do manuscrito. Paula Andréa de Melo Valença e Viviane Colares Soares de Andrade Amorim contribuíram na análise, na interpretação de dados e na redação e/ ou revisão do manuscrito.

\section{REFERÊNCIAS}

1. Santos CC, Ressel LB. Os adolescentes nos serviços de saúde. Adolesc Saúde [Internet]. 2013 [acesso em 2017 Ago 3];10(1):53-5. Disponível em: http://www.adolescenciaesaude.com/detalhe_artigo.asp?id=355

2. Centers for Disease Control and Prevention. Recommended immunizations for children 7-18 years. 2017 [acesso em 2017 Ago 3] Disponível em: https://www.cdc.gov/vaccines/schedules/easy-to-read/adolescent-easyread.html

3. Ministério da Saúde (BR). Nota informativa n³11 de 2016: CGPNI/DEVIT/SVS/MS. Brasília: Ministério da Saúde; 2016.

4. Ministério da Saúde (BR). Vacina de HPV é ampliada para meninos de 11 a 15 anos incompletos [Internet]. Portal Saúde. 2017 [acesso em 2017 Ago 3]. Disponível em: http://portalsaude.saude.gov.br/index.php/oministerio/principal/secretarias/svs/noticias-svs/28766-vacina-de-hpv-e-ampliada-para-meninos-de-11-a-15anos-incompletos-2

5. Sociedade Brasileira de Imunizações. Calendário de Vacinação SBI em Adolescente. São Paulo; 2016.

6. Levi GC. Recusa de vacinas: causas e consequências. São Paulo: Segmento Farma; 2013.

7. Ministério da Saúde (BR). Ministérios da Saúde e Educação se unem para vacinação nas escolas [Internet]. Brasília: Ministério da Saúde; 2017 [acesso em 2017 Mar 15]. Disponível em: http://portalsaude.saude.gov.br/ index.php/o-ministerio/principal/secretarias/svs/noticias-svs/27813-ministerios-da-saude-e-educacao-se-unempara-vacinacao-nas-escolas

8. Silva CS, Bodstein RCA. Referencial teórico sobre práticas intersetoriais em Promoção da Saúde na Escola. Ciênc Saúde Coletiva [Internet]. 2016 [acesso em 2017 Ago 3];21(6):1777-88. Disponível em: http://www.scielo. br/scielo.php?script=sci_arttext\&pid=S1413-81232016000601777\&lng=pt\&tlng=pt

9. Zhang SK, Pan XF, Wang SM, Yang CX, Gao XH, Wang ZZ, et al. Perceptions and acceptability of HPV vaccination among parents of young adolescents: a multicenter national survey in China. Vaccine [Internet]. 2013 [acesso em 2017 Ago 3];31(32):3244-9. Disponível em: http://dx.doi.org/10.1016/j.vaccine.2013.05.046

10. Gilkey MB, Calo WA, Moss JL, Shah PD, Marciniak MW, Brewer NT. Provider communication and HPV vaccination: the impact of recommendation quality. Vaccine [Internet]. 2016 [acesso em 2017 Ago 3];34(9):1187-92. Disponível em: http://dx.doi.org/10.1016/j.vaccine.2016.01.023

11. Coyne-Beasley T, Reiter PL, Liberty AC, Ford CA, Miles DR, Brewer NT. Awareness is not enoug: the need to increase meningococcal vaccine uptake clinical. Clin Pediatr (Phila) [Internet]. 2013 [acesso em 2017 Ago 3];52(5):441-50. Disponível em: http://journals.sagepub.com/doi/10.1177/0009922813481847

12. Mukherjee S, Madhivanan P, Li T, Albatineh A, Srinivas V, Jaykrishna P, et al. Correlates of completing routine vaccination among children in Mysore, India. J Infect Public Health [Internet]. 2015 [acesso em 2017 Ago 3];8(1):62-71. Disponível em: http://dx.doi.org/10.1016/j.jiph.2014.05.003

13. Seven M, Guvenl G, Sahin E, Akyuz A. Attitudes to HPV vaccination among parents of children aged 10 to 13 years. J Pediatr Adolesc Gynecol. 2015;28(5):382-6.

14. Bianco A, Pileggi C, lozzo F, Nobile CGA, Pavia M. Vaccination against human papilloma virus infection in male adolescents: knowledge, attitudes, and acceptability among parents in Italy. Hum Vaccin Immunother. 2014;10(9):2536-42. 
15. Reiter PL, Gupta K, Brewer NT, Gilkey MB, Katz ML, Paskett ED, et al. Provider-verified HPV vaccine coverage among a national sample of hispanic adolescent females. Cancer Epidemiol Biomarkers Prev. 2014;23(5):742-54.

16. Donahue KL, Stupiansky NW, Alexander AB, Zimet GD. Acceptability of the human papillomavirus vaccine and reasons for non-vaccination among parents of adolescent sons. Vaccine [Internet]. 2014 [acesso em 2017 Ago 3];32(31):3883-5. Disponível em: http://dx.doi.org/10.1016/j.vaccine.2014.05.035

17. Taylor JL, Zimet GD, Donahue KL, Alexander AB, Shew ML, Stupiansky NW. Vaccinating sons against HPV: results from a U.S. national survey of parents. PLoS One. 2014;9(12):1-11.

18. Friedrich HDA, Lizott LS, Kreuger MRO. Analysis of students' knowledge about human papillomavirus. DST J Bras Doenças Sex Transm. 2016;28(4):126-30.

19. Gottvall M, Larsson M, Höglund AT, Tydén T. High HPV vaccine acceptance despite low awareness among Swedish upper secondary school students HPV and upper secondary school students Gottvall et al. Eur J Contracept Reprod Health Care. 2009;14(6):399-405.

20. Kreuger MRO, Lizott LS, Friedrich H de A. Imunização contra HPV: nível de conhecimento dos adolescentes. Adolesc Saúde. 2017;14(3):38-45.

21. Vaidakis D, Moustaki I, Zervas I, Barbouni A, Merakou K, Chrysi MS, et al. Knowledge of Greek adolescents on human papilloma virus (HPV) and vaccination. Medicine (Baltimore) [Internet]. 2017 [acesso em 2017 Ago 3];96(1):1-7. Disponível em: https://www.ncbi.nlm.nih.gov/pubmed/28072683

22. Devroey D, Riffi A, Balemans R, Van De Vijver E, Chovanova H, Vandevoorde J. Comparison of knowledge and attitudes about vaccination between Belgian and immigrant adolescents. J Infect Public Health [Internet]. 2013 [acesso em 2017 Ago 3];6(1):1-9. Disponível em: http://dx.doi.org/10.1016/j.jiph.2012.10.005

23. Francisco PMSB, Donalisio MR, Gabriel FJO, Barros MBA. Vacinação contra hepatite B em adolescentes residentes em Campinas, São Paulo, Brasil. Rev Bras Epidemiol [Internet]. 2015 [acesso em 2017 Ago 3];18(3):552-67. Disponível em: http://www.scielo.br/scielo.php?script=sci_arttext\&pid=S1415-790X2015000300552\&lng=en\&tl $\mathrm{ng}=\mathrm{en}$

24. Giambi C, D'Ancona F, Del Manso M, De Mei B, Giovannelli I, Cattaneo C, et al. Exploring reasons for nonvaccination against human papillomavirus in Italy. BMC Infect Dis [Internet]. 2014 [acesso em 2017 Ago 3];14:1-9. Disponível em: http://www.pubmedcentral.nih.gov/articlerender.fcgi?artid=4233085\&tool=pmcentrez\&rendertyp $\mathrm{e}=$ abstract

25. Bowyer HL, Forster AS, Marlow LA V, Waller J. Predicting human papillomavirus vaccination behaviour among adolescent girls in England: results from a prospective survey. J Fam Plann Reprod Heal Care. 2014;40(1):14-22.

26. van Keulen HM, Otten W, Ruiter RAC, Fekkes M, van Steenbergen J, Dusseldorp E, et al. Determinants of HPV vaccination intentions among Dutch girls and their mothers: a cross-sectional study. BMC Public Health [Internet]. 2013 [acesso em 2017 Ago 3];13:1-21. Disponível em: http://www.pubmedcentral.nih.gov/articlerender.fcgi?art id $=3570492 \&$ tool $=$ mmcentrez\&rendertype $=$ abstrac

27. Mollers M, Lubbers K, Spoelstra SK, Weijmar-Schultz WC, Daemen T, Westra TA, et al. Equity in human papilloma virus vaccination uptake?: sexual behaviour, knowledge and demographics in a cross-sectional study in (un) vaccinated girls in the Netherlands. BMC Public Health. 2014;14:288.

28. Kester LM, Zimet GD, Forteeberry JD, Kahn JA, Shew ML. A national study of hpv vaccination of adolescent girls: rates, predictors, and reasons for non-vaccination. Matern Child Health J. 2014;17(5):879-85.

29. Choi HCW, Leung GM, Woo PPS, Jit M, Wu JT. Acceptability and uptake of female adolescent HPV vaccination in Hong Kong: a survey of mothers and adolescents. Vaccine [Internet]. 2013 [acesso em 2017 Ago 3];32(1):7884. Disponível em: http://dx.doi.org/10.1016/j.vaccine.2013.10.068

30. Reiter PL, Katz ML, Paskett ED. Correlates of HPV vaccination among adolescent females from appalachia and reasons why their parents do not intend to vaccinate. Vaccine. 2013;31(31):3121-5.

31. Shao SJ, Nurse C, Michel L, Joseph MA, Suss AL. attitudes and perceptions of the human papillomavirus vaccine in caribbean and african american adolescent boys and their parents. J Pediatr Adolesc Gynecol [Internet]. 2015 [acesso em 2017 Ago 3];28(5):373-7. Disponível em: http://dx.doi.org/10.1016/j.jpag.2014.11.003 
Endereço do primeiro autor:

Alisse Maria Chaves de Lima Peixoto

Universidade de Pernambuco - UPE

Av. General Newton Cavalcanti, 1650

Bairro: Tabatinga

CEP: 54753-220 - Camaragibe - PE - Brasil

E-mail: alissepeixoto@gmail.com

Endereço para correspondência:

Viviane Colares Soares de Andrade Amorim

Universidade de Pernambuco - UPE

Av. General Newton Cavalcanti, 1650

Bairro: Tabatinga

CEP: 54753-220 - Camaragibe - PE - Brasil

E-mail: viviane.colares@upe.br 